\title{
Combining Technology Mapping with Layout
}

\author{
MASSOUD PEDRAM ${ }^{\mathrm{a} *}$, NARASIMHA BHAT ${ }^{\mathrm{b}}$ and ERNEST S. KUH ${ }^{\mathrm{c}}$

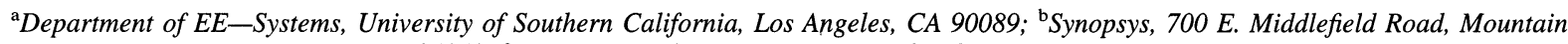

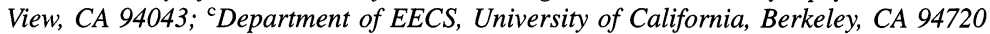

\begin{abstract}
Due to the significant contribution of interconnect to the area and speed of today's circuits and the technological trend toward smaller and faster gates which will make the effects of interconnect even more substantial, interconnect optimization must be performed during all phases of the design. The premise of this paper is that by increasing the interaction between logic synthesis and physical design, circuits with smaller area and interconnection length, and improved performance and routability can be obtained compared to when the two processes are done separately. In particular, this paper describes an integrated approach to technology mapping and physical design which finds solutions in both domains of design representation simultaneously and interactively. The two processes are performed in lockstep: technology mapping takes advantage of detailed information about the interconnect delays and the layout cost of various optimization alternatives; placement itself is guided by the evolving logic structure and accurate path-based delay traces. Using these techniques, circuits with smaller area and higher performance have been synthesized.
\end{abstract}

Keywords: Technology Mapping, Logic Decomposition, Placement, Layout-driven Logic Synthesis

\section{INTRODUCTION}

A principal goal of the electronic design automation effort is to provide designers with the capability to employ a complete top-down design methodology, compiling abstract architectural descriptions into an optimal implementation for a specific physical media. In fact, as density increases, system and ASIC designers have no choice but to converge to such a topdown design methodology. However, there is at least one major difficulty that must be overcome in order to develop the full potential and promise of this meth- odology. The problem is that the top-down design approach makes high-level decisions about optimization, scheduling, allocation, logic partitioning and restructuring, and so forth in terms of abstract views of behavior and structure. During these early steps, factors such as characteristics of the physical media, layout, interconnect and parasitics are ignored. Physical design which is expected to address these issues, comes much later in the design hierarchy. By then, many of the key architectural and structural decisions have been made, hence, limiting the capability of the physical design tools to generate the "best" solutions

*Corresponding author. 
in terms of area and performance. In addition, once some parameter at the physical design stage fails to satisfy a constraint imposed on it, the synthesis must be modified (even repeated) so as to accommodate the constraint. The new change may cause some other constraint to be violated and the process must be iterated.

It is, therefore, necessary to develop models, algorithms and techniques to increase the power and robustness of the top-down design methodologies. The goal-which is easy to state but difficult to achieve-is to integrate the various design steps keeping the computational complexity manageable. This paper is a step toward achieving this objective, that is, it describes novel techniques to couple logic synthesis to physical design and to explicitly control the interconnection length during logic synthesis. This will allow physical design and logic synthesis to evolve together and will ensure higher degrees of system level integration.

The goal of logic synthesis is to produce a circuit which satisfies a set of logic equations, occupies minimal silicon area and meets the timing constraints. Logic synthesis is often divided into a technologyindependent and a technology-dependent phase. In the first phase, transformations are applied on a Boolean network to find a representation with the least number of literals in the factored form. Additional timing optimization transformations are applied on this minimal area network to improve the circuit performance. The role of the technology-dependent phase is to finish the synthesis of the circuit by performing the final gate selection from a target library. The technology-dependent phase is, to a large extent, constrained by the structure of the optimized Boolean network. Most previous work in logic synthesis $[4,3]$ has focused on minimizing gate area and delay through a chain of gates without considering the area needed to hold the interconnect lines or the delay through the lines. It is generally assumed that interconnect optimization can be relegated to the physical design phase. Only recently some attention has been given to the interconnect optimization during logic synthesis $[1,20,19]$.

\subsection{Interconnect Effects}

Interconnections are becoming a major concern in today's high-performance, high-density ASIC designs because the distributed RC time delay of these lines increases rapidly as chip sizes grow and minimum feature sizes shrink [2]. With recent studies [23, 10] indicating that interconnections occupy more than half the total chip area and account for a significant part of the chip delay, it is appropriate that wiring is integrated into the cost function for logic synthesis. To elaborate on this point, consider Figure 1 which shows a performance-optimized two-input NAND gate driving a performance-optimized inverter gate through $0.2 \mathrm{~cm}$ of aluminum interconnect $(2 \mu \mathrm{m}$ wide, $0.5 \mu m$ thick, with a $1.0 \mu m$ thick field oxide beneath it). $0.2 \mathrm{~cm}$ is the expected length of a local interconnect line on a $2 \mathrm{~cm} \times 2 \mathrm{~cm}$ chip [2]. Two methods are used to calculate the rise time (to $50 \%$ of its final value) at the input of the inverter gate: one method ignores the capacitance and resistance of the interconnect line, the second method accounts for them [22]. Gate delays are taken from data sheets for an industrial 1-micron ASIC library; interconnect ca-

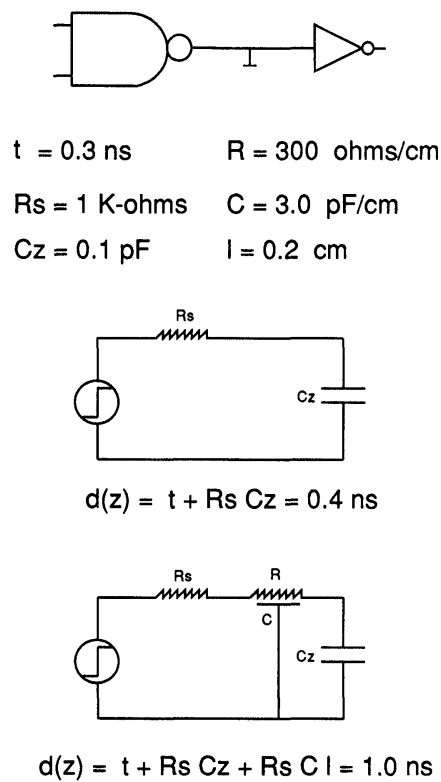

FIGURE 1 Comparing the interconnect delay to gate delay for 1-micron ASIC library. 
pacitance and resistance are calculated using expressions given in [2]. The delay calculations clearly show that interconnect capacitance dominates gate input capacitance and interconnect resistance may be ignored without introducing much error. When the $\mathrm{RC}$ tree forms branches, delays for the branching nodes can be calculated independently and accumulated to obtain the delays at the sink nodes. This calculation, however, requires knowledge of net topologies which is not available before global routing. This effect will not be addressed here. Furthermore, the transmission line properties of interconnect lines are ignored for on-chip connections. Therefore, an accurate expression for propagation delay through gates connected by local interconnect lines is given by

$$
d(z)=\tau+R_{s}\left(C_{z}+C l\right)
$$

where $\tau$ is the intrinsic gate delay, $R_{s}$ is the on-resistance of the driver gate, $C_{z}$ is the input capacitance of the fanout gate, $C$ is the interconnect capacitance per unit length and $l$ is the interconnect length.

In summary, with the existing technology, the capacitive term is dominated by the capacitance between the interconnection and substrate. For local aluminum lines, the resistive term is dominated by the on-resistance of the MOS transistor; for polysilicon and global aluminum lines on large-size circuits, the resistive term is controlled by the interconnection resistance. As the chip dimension increases and the minimum feature size decreases, the interconnection resistance increases rapidly while the MOS on-resistance remains relatively unchanged; the interconnection capacitance bottoms at about $1-2 \mathrm{pF} / \mathrm{cm}$ while the input gate capacitance decreases. Therefore, the distributed RC delay of interconnect lines will become even more dominant in the future.

\subsection{Concepts and Examples}

A Boolean network $\mathcal{N}$, is a directed acyclic graph (DAG) such that for each node in $\mathcal{N}$ there is an associated representation of a Boolean function $f_{i}$, and a
Boolean variable $y_{i}$, where $y_{i}=f_{i}$. There is a directed edge $(i, j)$ from $y_{i}$ to $y_{j}$ if $f_{j}$ depends explicitly on $y_{i}$ or $y_{i}$. A node $y_{i}$ is a fanin of a node $y_{j}$ if there is a directed edge $(i, j)$ and a fanout if there is a directed edge $(j, i)$. A node $y_{i}$ is a transitive fanin of a node $y_{j}$ if there is a directed path from $y_{i}$ to $y_{j}$ and a transitive fanout if there is a directed path from $y_{j}$ to $y_{i}$. Primary inputs are inputs of the Boolean network and primary outputs are its outputs. Intermediate nodes of the Boolean network have at least one fanin and one fanout. A Boolean network is an implementation or representation of a set of incompletely specified Boolean functions.

Given a Boolean network optimized by technology-independent logic operations and a target library, technology mapping is the process of binding nodes in the network to gates in the library such that the area of the final implementation is minimized, and timing constraints are satisfied. We illustrate the incorporation of the interconnect into technology mapping with a simple example. Figure 2a shows a small portion of a NAND-decomposed Boolean network. Source nodes $s_{i}$ have either been mapped (and hence have been assigned matching gates and positions) or are fixed at the circuit boundary. Note that $s_{1}$ and $s_{2}$ are positioned near one another but far from $s_{3}$ and $s_{4}$. The objective is to transfer the signals from $s_{i}$ 's to the sink node $t$ implementing the desired logic function while using minimum wire length. Conventional technology mappers attempt to find a solution with the smallest area gate which matches as many intermediate nodes as possible (the solution with one AND4 gate in Figure 2a). This is a good approach if the fanin gates $s_{i}$ can be placed near the matching gates. However, in many cases, these gates are either strongly connected to different gate clusters on the layout plane or are fixed at the circuit boundary and hence may have positions far from one another and from the matching gate. Therefore, a solution with one distribution point may incur a large interconnection cost. In fact, there is often an optimum number of mapped gates greater than one which will result in overall minimum wire cost as depicted in Figure 2. If the number of sources is large, say four or more, then 


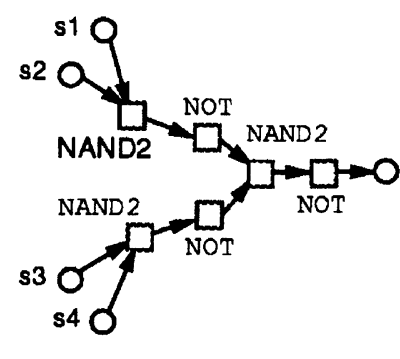

(1)

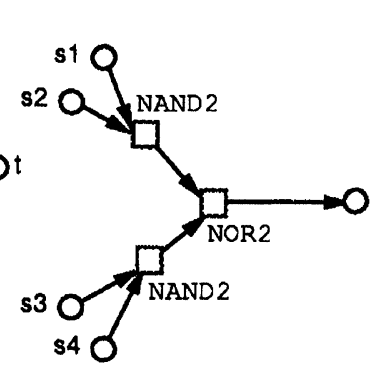

(2)

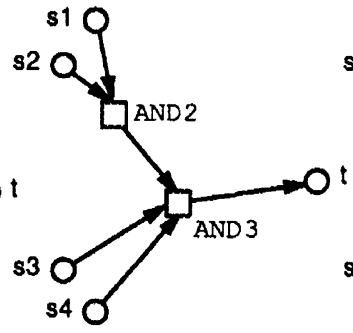

(3)

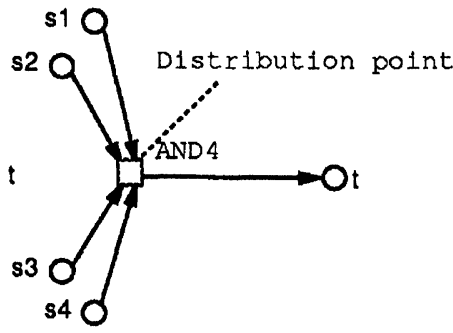

(4)

(a)

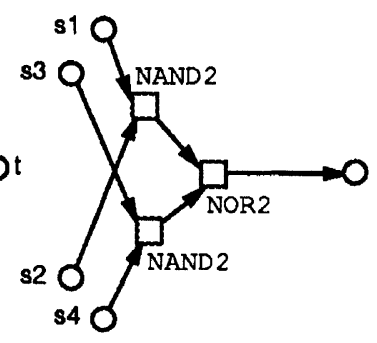

(2)

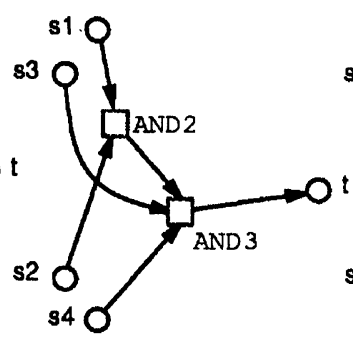

(3)

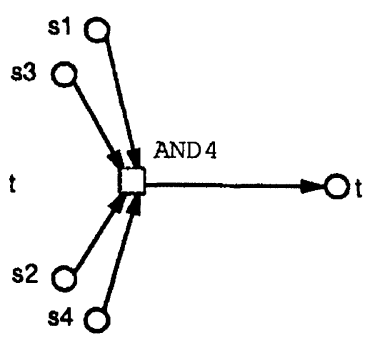

(4)

(b)

FIGURE 2 Active gate area versus wire length trade-off.

it will pay off to consider how close the sources can be placed by a good placement optimizer before deciding whether a solution of one gate (with high fanin count) or a solution of more than one gate (with low fanin counts) should be chosen. The technology mapper proposed here selects the solution with one AND2 and one AND3 gate in Figure 2a.

Figure $2 \mathrm{~b}$ illustrates the importance of a layoutdirected technology decomposition for the technology mapping scheme. This figure shows the same decomposition tree as in Figure 2a. However, this time as a result of placing the NAND-decomposed network, source nodes $s_{1}$ and $s_{3}\left(s_{2}\right.$ and $\left.s_{4}\right)$ have been positioned near one another. Signals coming from $s_{1}$ and $s_{3}\left(s_{2}\right.$ and $\left.s_{4}\right)$ enter the network at topologically distant points. This is undesirable because the mapper has lost the option of reducing the wiring cost by breaking one big gate into smaller gates, i.e., in Fig- ure $2 b$, the mapping solution with one AND4 gate is superior to other solutions in terms of both total gate area and interconnection length. Therefore, Figure 2a provides better technology decomposition (and hence potential for higher quality mapping) than that in Figure $2 b$.

\subsection{Overview}

In this paper, we present LDTM [20], a technology mapping program, built on top of MIS [4], which tightly and interactively couples mapping to placement. LDTM's key idea is to generate a placement of the optimized multi-level Boolean network which captures the structure of the network. The placement information is used to evaluate the cost of a gate matching during decomposition and mapping pro- 
cesses. The placement is dynamically updated in order to maintain the correspondence between logic and layout representations. In the end, a mapped network along with a companion placement solution are generated. The placement solution is then globally relaxed in order to produce a feasible placement according to the target layout style (e.g., standard-cell or sea-of-gates). This design flow is in contrast with the existing synthesis systems which separate technology mapping and placement steps.

Technology Mapping is driven by layout information derived from the placement of the Boolean network. It is, therefore, essential to generate a placement solution which not only captures the global connectivity structure of the network, but also produces the shortest directed path between any pair of primary input-primary output nodes. [16] describes a flow-oriented approach to the placement of general directed acyclic graphs.

In Section 2, the technology mapping paradigm is presented. Section 3 describes a technique for creating a feasible placement solution from the companion placement solution. Sections 4 and 5 contain experimental results, discussions and future research directions.

\section{LAYOUT-DRIVEN TECHNOLOGY MAPPING}

A successful and efficient solution to the technology mapping problem was suggested by K. Keutzer and implemented in DAGON [12] and MIS [9]. The idea is to reduce technology mapping to DAG covering and to approximate DAG covering by a sequence of tree coverings which can be performed optimally using dynamic programming. DAGON and MIS technology mappers generate circuits with small active cell area but ignore area contributed by interconnections between gates. Consequently, these mappers produce gates with high fanin count which often increase routing congestion during the final layout and increase interconnection lengths. Similarly, performance-oriented technology mapping programs suffer from lack of detailed information about the interconnect delay. The approach presented here, however, explicitly considers interconnect and routing complexity during the mapping.

Our layout-directed technology mapping is based on the DAG covering formulation which can be summarized as follows. A set of complete base functions is chosen, such as a two-input NAND gate and an inverter. The optimized logic equations (obtained from technology independent optimization) are converted into a graph where every node is one of the base functions. This graph is called the subject graph. Each library gate is also represented by a graph consisting of only base functions. Each such graph is called a pattern graph. A library gate may have many different pattern graphs. The technology mapping problem is then defined as the problem of finding a minimum cost covering of the subject graph by choosing from the collection of pattern graphs for all gates in the library. For area optimization, the cost of a cover is defined as the sum of gate areas. For performance optimization, the cost of a cover is defined as the critical path delay of the resulting circuit. In particular, the mapper binds a given logic circuit onto a set of gates in the target library minimizing postlayout area, delay or area under delay constraints.

\subsection{Technology Decomposition}

The procedure for converting an optimized Boolean network into the subject graph (i.e., technology decomposition) is not unique, and it is an open problem to determine which of the possible subject graphs yields an optimum solution when an optimum covering algorithm is applied [5]. The goal of our technology decomposition procedure is to find a circuit representation with minimum signal arrival time at the primary outputs and minimum number of wire crossings (given an initial placement of the optimized Boolean network).

The decomposition process starts by constructing AND-OR trees implementing the sum-of-product representation of the logic function associated with each intermediate node in the Boolean network. The 
function of AND subtrees is to compute the product terms (cubes) and that of the OR subtrees is to compute the sum of the product terms. The input signals to the AND subtrees and then the cubes in the OR subtrees are ordered. The conversion from the ordered AND-OR subtrees to the gates in base function set is accomplished using unbalanced NAND decomposition by providing late arriving inputs with shorter paths through the NAND-decomposed subnetwork $[25,17]$.

In order to derive the input signal ordering, one refers to the companion placement solution for the Boolean network. Each multi-pin net signal is modeled by a star connection from the source toward the sinks. By circularly traversing around each node (for example, starting from the positive horizontal axis and proceeding in a counter-clockwise fashion), a unique ordering of the input signals to the node can be determined. This ordering is directly related to the positions of the fanin nodes with respect to the node in question.

The cube ordering is achieved by setting up a linear assignment problem. $S$ slots are placed on an imaginary inner circle around the node, and the projections of the fanin signals into an imaginary outer circle around the node are found (Figure 3 ). Then, a

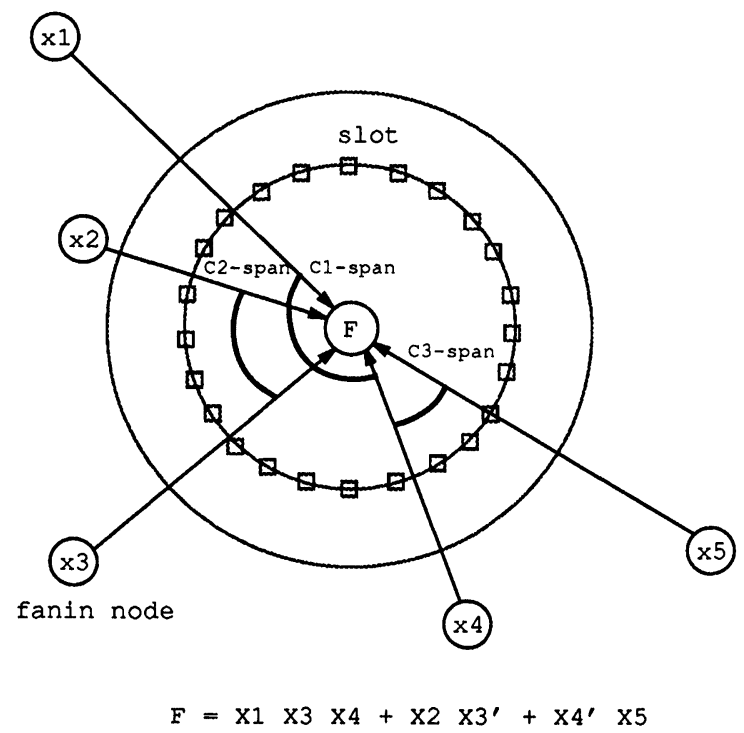

FIGURE 3 Cube ordering viewed as a linear assignment problem. linear assignment cost matrix $C$ is set up whose $c_{i k}$ entry corresponds to the cost of assigning cube $i$ to slot $k$. This entry is equal to zero if slot $k$ falls inside the shortest circular span for the immediate support of cube $i$. Otherwise, the cost is proportional to the angular distance of slot $k$ from the nearest end of the support span of cube $i$. The linear assignment program [7] determines a cube assignment with the minimum sum-cost. The cube ordering is easily derived from the cube positions obtained by the above linear assignment procedure. The process of ordering input signals, cubes and then primitive gate decomposition is recursively applied to all nodes in the Boolean network in order to produce the subject graph.

\subsection{DAG Covering for Minimum Layout Area}

Consider a Boolean network $N$, which has been transformed into a subject graph consisting of only twoinput NAND and inverter gates. This is the network in its unmapped form which will be referred to as the inchoate network, $N_{\text {inchoate }}$. In DAGON, $N_{\text {inchoate }}$ is partitioned into a set of maximal trees, $T_{i}$, and an optimal dynamic programming solution is found for each tree. In MIS, $N_{\text {inchoate }}$ is split into a set of logic cones $K_{i}$, where each cone corresponds to a primary output and all its transitive fanin nodes. This allows covering across tree boundaries and, as a result, may duplicate logic. The MIS technology mapper implements DAGON as a subset. Our mapper uses cone partitioning.

Assume that the cost of match $m$ at node $v$ is to be calculated (Figure 4). This cost consists of two (linearly combined) terms:

$$
\begin{gathered}
\text { area_cost }(v, m)=\operatorname{area}(\text { gate }(m)) \\
+\sum_{v_{i} \in \text { inputs }(v, m)} \operatorname{area} \operatorname{cost}\left(v_{i}, m_{i}\right) \\
\text { wire_cost }(v, m)=\text { wire }\left(\operatorname{gate}(m), \text { gate }\left(v_{i}\right)\right) \\
+\sum_{v_{i} \in \text { inputs }(v, m)} \text { wire_cost }\left(v_{i}, m_{i}\right) .
\end{gathered}
$$

where $\operatorname{inputs}(v, m)$ refers to the list of nodes of $N$ which correspond to the inputs of $m$. gate $(m)$ is the 


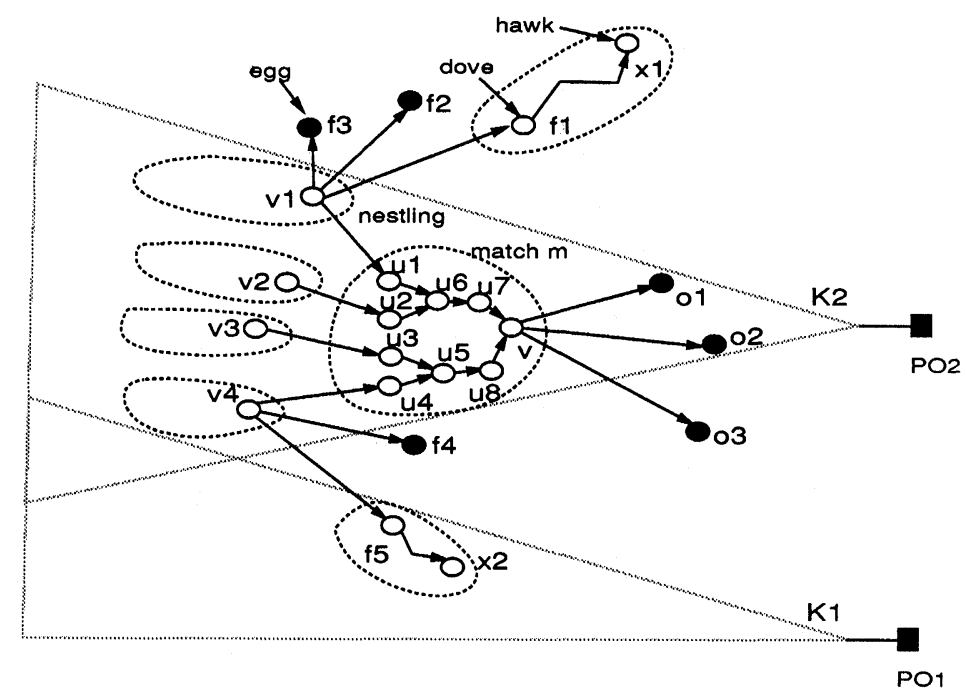

FIGURE 4 Cost calculation for a candidate match.

physical gate corresponding to $m$. gate $\left(v_{i}\right)$ is the best gate matching at node $v_{i}$. The area cost calculation is similar to that in MIS. The wire cost wire_cost $(v, m)$ consists of two terms. The first term is the interconnection length required to complete connections from gate $(m)$ to its fanin gates, i.e., gate $\left(v_{i}\right)$. The second term is the dynamic programming recursive cost and represents the sum of wire lengths required to connect all gates from primary inputs up to $\operatorname{gate}\left(v_{i}\right)$. In order to calculate wire_cost $(v, m)$, the position of $m$ must be known as shown next.

At the beginning, nodes are assigned valid placePositions based on the initial global placement solution. As nodes are mapped, mapPositions are calculated and stored on nodes. In particular, match $m$ is placed at the center of mass (or median) of its fanin and fanout rectangles. Due to the postorder traversal of the network during the mapping procedure, in$\operatorname{puts}(v, m)$ have already been mapped and therefore their mapPositions are used; outputs(v) are not mapped yet and their placePositions are used.

Depending on the wire length metric adopted, the local placement problem can be solved efficiently or can become difficult. Consider Figure 5, which shows the enclosing rectangles for the fanin and fanout nets of match $m$ at $v .^{2}$ Given a norm and the coordinates of these fanin and fanout rectangles $r$, the problem is to find a point $p$ which results in the minimum sum of distances between that point and the rectangles. In case of the Manhattan norm, the solution easily follows by observing that the distance function has a separable form with respect to the variables $x$ and $y$. That is, the $x$ distance of point $p$ from rectangle $r$ is $1 / 2(|r . l l . x-p . x|+|r . u r . x-p . x|-|r . u r . x-r . l l . x|)$ where $l l$ and $u r$ refer to the lower left and the upper right of rectangle $r$. The constant term is dropped and the problem can be restated as: Find $x$ such that $\Sigma_{i} \mid x_{i}$ $-x \mid$ is minimum where $x_{i}$ corresponds to either the left or the right corner point coordinates of each of the rectangles. The problem is a special case of solving for the median of a graph which is presented in [11]. It can be shown that this problem, treating only a linear tree rather than a general graph, is very easy to solve; the solution is the median point for the sorted list of $x_{i}$ 's. For the Euclidean norm, the optimal point location problem can be solved approxi-

\footnotetext{
${ }^{1}$ The enclosing rectangle for a fanin (fanout) net is the smallest rectangle enclosing the gates connected by the net.
} 


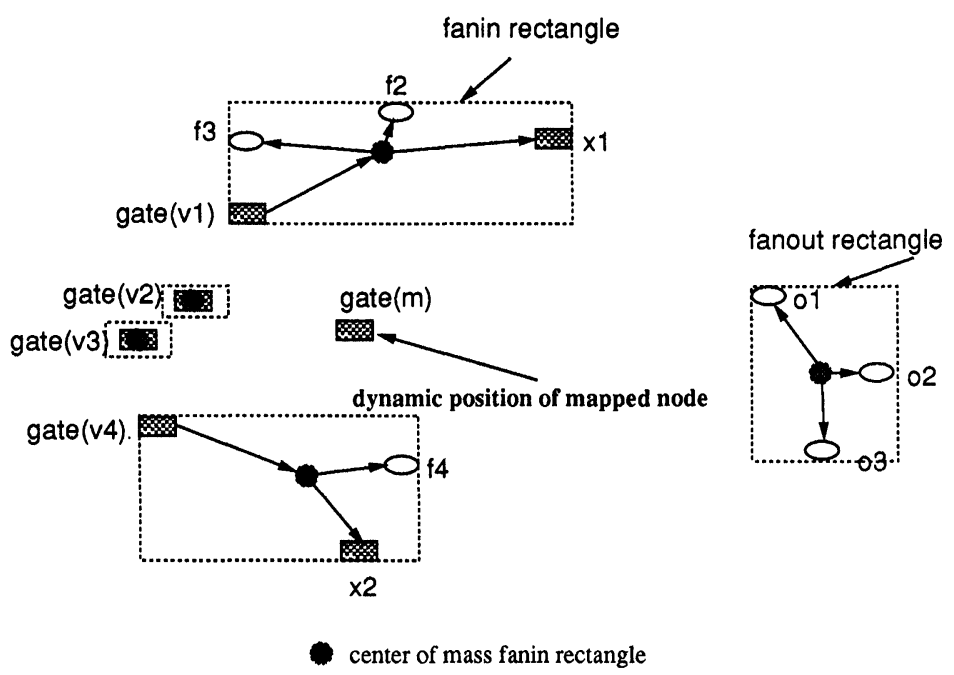

FIGURE 5 Dynamic updating of placement positions (Euclidean norm).

mately by placing $m$ at the center of mass of its fanin and fanout rectangles [18].

When constructing the enclosing rectangle of fanin net $i$, it is important to know fanout nodes of source node $v_{i}$. These fanout nodes of $v_{i}$ are dynamically defined based on the current partially mapped network. First, we give some definitions. A sink node in a pattern graph is defined as a node which does not fanout to any other node in the pattern graph. In Figure 4, assume that cone $K_{1}$ has been mapped. At this point, nodes can be classified into four categories. An egg is a node which has not been processed (visited) by the mapper. A nestling is a node in the current cone, $K_{2}$, which has been visited. It cannot be predicted whether a nestling will be present in the final mapped network until primary output $\mathrm{PO}_{2}$ is reached. A dove is a node in $K_{1}$ which is a non-sink element of some pattern match. Such a node will not be present in the final mapped network because it has been merged into another. A hawk is a node in $K_{1}$ which is a sink node in some pattern match. Such a node will inevitably show up in the final mapped network. Note that every dove has been merged into (fallen prey to) at least one hawk. A nestling can become a hawk or a dove. Due to the possibility of logic duplication, it may be possible for a dove to reincarnate and restart the node's life cycle as an egg and later become a hawk. The dynamic fanout of fanin $v_{i}$ for match $m$ at $v$ is a hawk, a nestling or an egg which has $v_{i}$ as its fanin. For example, the list of dynamic fanouts of node $v_{4}$ consists of nodes $v, x_{2}$ and $f_{4}$. During the construction of the enclosing rectangle of net $i$ (say 4 ), nodes covered by the current match are excluded from the net. In addition, mapPositions are used for hawks and nestlings ( $x_{2}$ and $v_{4}$ ) and placePositions are used for eggs $\left(f_{4}\right)$.

After positioning gate $(m)$, the wire cost associated with the matching of $m$ at node $v$ must be calculated. This cost consists of the sum of the wire lengths from $m$ to its fanins. Consider fanin $v_{i}$ of $m$. If it is driving only the input pin of $m$, the wire length calculation is point-to-point. However, if it is driving multiple fanout pins (including input pin of $m$ ), then the half perimeter length of the minimum box bounding all pins on the net is used. Note that during technology mapping for minimum area, only length of the line connecting each fanin $v_{i}$ to $m$ is of interest. Therefore, the net length must be divided by the dynamic fanout count at $v_{i}$ in order to get the expected wire length contributed by connection from $v_{i}$ to $m$ and thereby avoid duplicate accounting of the wire cost.

\subsection{DAG Covering for Minimum Circuit Delay}

In the delay mode, the best mapping at a node is determined based on the arrival time of the signal at the node output. As pointed out earlier, it is the delay 
in the interconnect that is of prime importance. Hence, it is only natural that wiring delay is incorporated into the calculation of the arrival time during technology mapping.

Consider a gate $g$ with output line $y$ and input lines $i, i=1 \ldots p$. Let $g$ fanout to inputs of $g_{j}$. In a simple linear delay model, the delay through $g$ is a linear function of its output load capacitance $C_{L}$. The slope of this linearity can be thought of as the output resistance and the offset (at zero $C_{L}$ ) can be thought of as the intrinsic delay through $g$. In general, the delays from different inputs to the output are different. Therefore, the intrinsic delay from input $i$ to $y$ is denoted by $I_{i}$, and the output resistance at $y$ corresponding to input $i$ is denoted by $R_{i} . I_{i}$ and $R_{i}$ each have separate values for rising and falling delays.

Based on this general model, the arrival time at $y$ from input $i, t_{y i}$, can be easily calculated as $t_{y i}=t_{i}+$ $I_{i}+R_{i} C_{L}$ where $t_{i}$ is the arrival time at input line $i$. Using a worst case analysis, the output arrival time at $y, t_{y}$ is defined as the time at which all signals from input lines $i$ will be available at $y$ and is given by $t_{y}=$ $\max \left\{t_{y i}\right\}$ computed over all $i, i=1 \ldots p$. This calculation for the arrival time requires that the value of $C_{L}$ be known. $C_{L}$ is the equivalent capacitive load at $y$. This capacitance is modeled as $C_{L}=\sum_{j=1}^{n} C_{j}+C_{w}$ where $C_{j}$ denotes the capacitance at the input of fanout gate $g_{j}$, and $n$ is the number of fanout nodes. $C_{w}$ represents the capacitance due to the interconnections which connect $g$ to its fanout nodes. We calculate $C_{w}$ accurately using the placement information which provide the horizontal and vertical extents of the signal nets and using a technology file which provides the capacitance per unit length of horizontal and vertical interconnect.

In Figure 4, fanouts of $v$ are not yet mapped. This implies that the load $C_{L}$, at the output of gate $(m)$ cannot be determined exactly. This difficulty can be handled by assuming a default load, i.e., all types of gates are assumed to have the same input capacitance. This assumption is also adopted in MIS2.1. However, in order to calculate the wiring capacitance, positions of gates at the fanouts of $v$ must be known. This information is not available and instead positions of the fanout gates are read from the initial placement solution of the subject graph. The simpli- fication gives rise to inaccuracies in the arrival time calculation. To prevent the inaccuracy from propagating through, the following observation is used: When matching $m$ at $v$, the capacitance at the output of inputs $(v, m)$ is known because the type and position of their fanout gate, which is gate $(m)$, is known. If the output arrival times of $\operatorname{inputs}(v, m)$ are updated, then the input arrival time of gate $(m)$ is accurate. Therefore, the output arrival time for gate $(m)$ can be calculated with less error (that is, error will be due to the unknown load only).

\section{PLACEMENT RELAXATION}

After the logic synthesis stage, a net list of gates and a companion placement solution are available. The placement solution, however, has overlapping gates and has not yet been mapped to rows (in the case of the standard cell layout methodology) or to slots (in the case of the sea-of-gates style). The objective of global relaxation step is to eliminate gate overlaps and produce an even distribution of gates over the layout image. Two basic approaches are generally used for mapping a global placement result to legal locations: (1) Perform a minimum squared error linear assignment which maps the cells in the global placement to the legal positions simultaneously; (2) Use a hierarchical bi-partitioning technique to obtain a feasible placement solution.

In our application, the initial (globally optimized) placement is modified throughout the synthesis (based on local considerations only), and therefore, the resulting companion placement is not globally optimized. However, we do not want to throw away the companion placement (which has influenced many of our decisions during the synthesis) and place the mapped network from scratch.

We have adopted the top-down bi-partitioning heuristic in the following way. Our placement procedure consists of alternating and interacting global function optimization and partitioning steps. In particular, for a circuit with $M$ gates, the placement procedure goes through $m=\left\lceil\log _{2} M\right\rceil$ steps in order to produce a detailed placement. Now, assume that an initial place- 
ment solution for the circuit and two parameters $N_{s}$ and $N_{f}$ are given. These parameters specify the start and finish conditions for the relaxation procedure, that is, relaxation begins when number of modules per hierarchical region is $N_{s}$ and ends when this number is $N_{f}$. Let $s=\left\lceil\log _{2} N_{s}\right\rceil, t=\left\lceil\log _{2} N_{f}\right\rceil$, then $m \geq s$ $\geq t \geq 0$. We modify the placement procedure so that it goes through steps $m-s, \ldots, m-t$ only, thereby, achieving the relaxation goal without drastically disturbing the initial placement solution. Note that $N_{s}=$ $M$ corresponds to doing placement from scratch while $N_{f}=1$ corresponds to the detailed placement (one module per region). We have obtained the best results when setting $N_{s}=M / 8$ and $N_{f}=1$.

\section{EXPERIMENTAL RESULTS}

Our objective was to show that by integrating technology mapping and gate placement, one can improve the quality of mapping both in terms of layout area and circuit performance. In order to provide a fair basis for comparison, two pipelines were used to produce the results: 1) Read in the optimized circuit; do balanced tree technology decomposition; read in the lib2.genlib standard cell library; run MIS technology mapper in timing mode; write the mapped circuit to the database; do detailed placement and routing. 2) Read in the optimized circuit; do layout driven technology decomposition; read in the lib2.genlib standard cell library; run LDTM in timing mode; do detailed placement and routing. The following tools were used for generating the layouts: the GORDIAN package for placement [14], the TIMBER WOLF global router [15], and the YACR detailed router [21].

In both cases, the technology-independent optimizations were performed using the MIS program. The benchmarks were optimized for minimum area using the rugged script [24]. This script produces the optimized circuits. The literal count results (before technology mapping) are listed in Table I.

Table II shows post-mapping comparisons between the MIS and LDTM in terms of total gate area and logic delay calculated by ignoring the wiring loads (i.e., using the input capacitances, the intrinsic and fanout delays for logic gates only), and the cpu times
TABLE I Multi-level benchmarks: number of literals in factored form

\begin{tabular}{lrc}
\hline Ex. & original & optimized \\
\hline 9symml & 277 & 183 \\
C1355 & 1032 & 552 \\
C1908 & 1497 & 535 \\
C3540 & 2934 & 1283 \\
C432 & 372 & 219 \\
C5315 & 4369 & 1763 \\
C880 & 703 & 414 \\
apex6 & 904 & 732 \\
apex7 & 289 & 243 \\
b9 & 236 & 124 \\
duke2 & 763 & 452 \\
f51m & 169 & 80 \\
rot & 764 & 664 \\
z4ml & 77 & 43 \\
\hline
\end{tabular}

on a Sparc Station II workstation. The MIS mapper produces somewhat better results in terms of logic delay and is faster by a factor of 1.8 .

Table III shows post-placement comparisons between the MIS and LDTM in terms of total chip area and circuit delay calculated after placement and routing and accounting for the wiring loads. As expected, LDTM shows an average chip area improvement of $7 \%$ and a total delay improvement of $9 \%$ compared to MIS. This improvement is mainly due to reduced wiring load on critical signal paths as a result of LDTM's gate selection policy. We used a value of 3 $\mathrm{pF} / \mathrm{cm}$ for the capacitance per unit length. As the technology scales down and the contribution of the wiring delay to total circuit delay increases, the percentage improvement of a layout-driven mapper (such as LDTM) over a conventional mapper (such as that of MIS) will increase.

These tables were generated by running the MIS and LDTM mappers in the timing mode. A similar trend existed when we ran these mappers in the area mode.

\section{CONCLUDING REMARKS}

In this paper, we put forth techniques for coupling logic synthesis and placement. To achieve this objective, we studied the effects of interconnect on circuit 
TABLE II Comparison of the total gate area and logic delay after placement and routing. The cpu time reflects the mapping time

\begin{tabular}{|c|c|c|c|c|c|c|}
\hline \multirow[t]{2}{*}{ example } & \multicolumn{3}{|c|}{ MIS } & \multicolumn{3}{|c|}{ LDTM } \\
\hline & $\begin{array}{c}\text { gate area } \\
\mathrm{mm}^{2}\end{array}$ & $\begin{array}{c}\text { logic delay } \\
n s\end{array}$ & $\begin{array}{c}\text { cpu time } \\
s\end{array}$ & $\begin{array}{c}\text { gate area } \\
\mathrm{mm}^{2}\end{array}$ & $\begin{array}{c}\text { logic delay } \\
n s\end{array}$ & $\begin{array}{c}\text { cpu time } \\
s\end{array}$ \\
\hline 9symml & 0.288 & 16.19 & 9.4 & 0.280 & 16.23 & 15.2 \\
\hline C1355 & 0.612 & 23.77 & 36.1 & 0.664 & 23.94 & 48.4 \\
\hline C1908 & 0.830 & 32.32 & 59.3 & 0.811 & 33.01 & 74.5 \\
\hline C 3540 & 1.810 & 46.92 & 92.7 & 1.933 & 47.19 & 192.3 \\
\hline $\mathrm{C} 432$ & 0.374 & 28.09 & 24.9 & 0.400 & 27.56 & 38.5 \\
\hline C5315 & 2.300 & 37.10 & 75.6 & 2.305 & 40.35 & 181.5 \\
\hline $\mathrm{C} 880$ & 0.637 & 32.85 & 33.4 & 0.640 & 33.10 & 51.8 \\
\hline apex6 & 1.005 & 15.28 & 25.7 & 1.012 & 15.06 & 56.9 \\
\hline apex 7 & 0.342 & 14.20 & 10.9 & 0.344 & 14.31 & 27.1 \\
\hline b9 & 0.187 & 5.84 & 5.2 & 0.189 & 6.25 & 8.8 \\
\hline duke2 & 0.655 & 16.28 & 30.3 & 0.670 & 17.63 & 51.5 \\
\hline $\mathrm{f} 51 \mathrm{~m}$ & 0.120 & 22.15 & 3.9 & 0.116 & 22.37 & 5.7 \\
\hline rot & 0.918 & 20.73 & 26.7 & 0.919 & 21.98 & 59.5 \\
\hline $\mathrm{z} 4 \mathrm{ml}$ & 0.080 & 10.11 & 3.1 & 0.087 & 10.21 & 4.5 \\
\hline
\end{tabular}

area and performance, presented appropriate models and computational procedures for estimating wiring delay during synthesis, and introduced a scheme for maintaining simultaneous and interactive data representations in logic and layout domains.

Layout information should be considered during all logic synthesis operations in order to improve the circuit routability and reduce the interconnect contribution to circuit area and delay. This is important since, as discussed earlier, interconnects play a pri- mary role in determining the longest paths through a circuit. Therefore, layout-driven algorithms and techniques for performing the various logic operations must be developed.

In manipulating the initial representation of the logic function, five operations are key: decomposition, extraction, factoring, substitution, and collapsing [5]. We are developing layout-integrated procedures for these logic operation which aim at minimizing the total interconnection length of the synthesized

TABLE III Comparison of the final chip area and circuit delay after placement and routing. The cpu time reflects the mapping, placement and routing times

\begin{tabular}{|c|c|c|c|c|c|c|}
\hline \multirow[t]{2}{*}{ example } & \multicolumn{3}{|c|}{ MIS } & \multicolumn{3}{|c|}{ LDTM } \\
\hline & $\begin{array}{c}\text { chip area } \\
m^{2}\end{array}$ & $\begin{array}{c}\text { total delay } \\
n s\end{array}$ & $\underset{s}{\text { cpu time }}$ & $\begin{array}{c}\text { chip area } \\
\mathrm{mm}^{2}\end{array}$ & $\begin{array}{c}\text { total delay } \\
n s\end{array}$ & $\begin{array}{c}\text { cpu time } \\
s\end{array}$ \\
\hline 9symml & 0.860 & 22.66 & 32.9 & 0.841 & 21.15 & 40.1 \\
\hline C1355 & 2.371 & 36.16 & 131.2 & 1.883 & 31.21 & 154.4 \\
\hline C1908 & 3.233 & 47.73 & 150.9 & 2.764 & 43.77 & 161.1 \\
\hline C 3540 & 8.224 & 70.26 & 296.4 & 8.106 & 65.01 & 420.8 \\
\hline $\mathrm{C} 432$ & 1.197 & 37.46 & 67.2 & 1.101 & 34.84 & 82.1 \\
\hline C5315 & 10.754 & 60.85 & 776.1 & 9.522 & 55.66 & 861.0 \\
\hline $\mathrm{C} 880$ & 1.961 & 45.50 & 126.1 & 1.772 & 41.93 & 144.2 \\
\hline apex6 & 3.887 & 27.43 & 375.0 & 3.794 & 25.19 & 397.6 \\
\hline apex 7 & 0.935 & 18.96 & 73.9 & 0.906 & 15.70 & 84.5 \\
\hline b9 & 0.491 & 8.03 & 42.1 & 0.486 & 7.52 & 45.8 \\
\hline duke2 & 2.604 & 25.25 & 100.9 & 2.556 & 23.19 & 120.1 \\
\hline $\mathrm{f} 51 \mathrm{~m}$ & 0.303 & 29.34 & 17.1 & 0.267 & 27.67 & 18.6 \\
\hline rot & 3.268 & 30.88 & 387.2 & 3.007 & 27.82 & 418.3 \\
\hline $\mathrm{z} 4 \mathrm{ml}$ & 0.179 & 12.72 & 13.0 & 0.170 & 11.90 & 14.6 \\
\hline
\end{tabular}


network as well as the total number of literals in the factored form representation of the network. For example, consider the extraction procedure which identifies common subexpressions among various functions. The literal value of a kernel measures the difference in the number of literals in the network if that rectangle is extracted and made into a new node [6, 4]. We can similarly define the interconnection value of a kernel as the difference in the total wire length in the network if that kernel is extracted and made into a new node. We then use a kernel-selection policy which chooses a kernel with the greatest cost reduction in terms of a linear combination of literal and interconnection values [19].

Performance optimization logic restructuring operations (e.g., depth reduction [13], partial collapse and resynthesis along the critical paths [25], logic clustering and partial collapse [26], etc) are often used to speed the synthesized network. Layout information can be exploited by most of these transformations. For example, consider circuit speed-up procedure given in [25]. This procedure identifies an $\epsilon$-network (i.e., a sub-network in which all the signals have a slack within $\epsilon$ of the most negative slack), partially collapses the nodes in the $\epsilon$-network, and redecomposes these nodes using a timing-driven decomposition scheme such that the resulting network is faster, and the area increase is minimal. During this process, if information about the interconnect delay becomes available (through a placement of the original network and incremental calculation of positions for the collapsed and later newly created nodes), then the following improvements are possible: the timing analysis for finding the $\epsilon$-critical paths is performed more accurately; during decomposition both literal saving and interconnect saving values of the candidate divisors are considered; interconnect delays influence the selection of the best timing-divisors; and NAND-decomposition of collapsed nodes can be performed using information about positions of fanin nodes.

The layout-driven technology mapping procedure can be easily extended to solve the problem of minimizing gate area plus wiring subject to required time constraints on the primary outputs. The idea is to incorporate the wiring area and delay into the initial calculation of area-delay trade-off curves and the subsequent gate selection steps [8].

Previous approaches for Table Look-Up (TLU) based FPGA's have aimed at minimizing the number of TLU's. However, routing resources in these architectures are very limited and therefore mapping for improved routability is an important consideration. The layout-driven approach can be extended to the FPGA synthesis problem.

\section{Acknowledgements}

This work was sponsored in part by the National Science Foundation and by the Semiconductor Research Corporation.

\section{References}

[1] P. Abouzeid, K. Sakouti, G. Saucier, and F. Poirot. Multilevel synthesis minimizing the routing factor. In Proceedings of the 27th Design Automation Conference, pages 365368, June 1990.

[2] H. B. Bakoglu. Circuits, interconnections, and packaging for VLSI. Addison-Wesley, 1990.

[3] K. Bartlett, D. Bostick, G. Hachtel, R. Jacoby, M. Lightner, P. Moceyunas, C. Morrison, and D. Ravenscroft. BOLD: A multiple-level logic optimization system. In Proceedings of the IEEE International Conference on Computer Aided Design, 1987.

[4] R. Brayton, R. Rudell, A. Sangiovanni-Vincentelli, and A. Wang. MIS: A multiple-level logic optimization system. IEEE Transactions on Computer-Aided Design of Integrated Circuits and Systems, CAD-6(6), Nov. 1987.

[5] R. K. Brayton, G. D. Hachtel, and A. L. Sangiovanni-Vincentelli. Multilevel logic synthesis. In Proceedings of the IEEE, volume 78, pages 264-300, February 1990.

[6] R. K. Brayton. Algorithms for multilevel logic synthesis and optimization. In P. Antognetti, G. DeMicheli, and A. Sangiovanni-Vincentelli, editors, NATO ASI on Logic Synthesis and Silicon Compilation for VLSI. Kluwer, Dordrecht, The Netherlands, 1987.

[7] R. E. Burkhard and U. Derigs. Assignment and matching problems: solution methods with Fortran programs. Springer Verlag, 1980.

[8] K. Chaudhary and M. Pedram. A near-optimal algorithm for technology mapping minimizing area under delay constraints. Proceedings of the 29th Design Automation Conference, June 1992.

[9] E. Detjens, G. Gannot, R. Rudell, A. Sangiovanni-Vincentelli, and A. Wang. Technology mapping in MIS. In Proceedings of the IEEE International Conference on Computer Aided Design, pages 116-119, November 1987.

[10] Y. A. El-Mansy and W. M. Siu. MOS technology advances. In G. Rabbat, editor, Handbook of Advanced Semiconductor Technology and Computer Systems, pages 229-259. Van Nostrand-Reinhold, Princeton, New Jersey, 1988. 
[11] S. L. Hakimi. Optimum locations of switching centers and the absolute centers and medians of a graph. Operations Research, 12:450-459, 1964.

[12] K. Keutzer. DAGON: Technology mapping and local optimization. In Proceedings of the Design Automation Conference, pages 341-347, June 1987.

[13] K. Keutzer and M. Vancura. Timing optimization in a logic synthesis system. In Proceedings of the International Workshop on Logic and Architectural Synthesis, May 1987.

[14] J. M. Kleinhans, G. Sigl, F. M. Johannes, and K. J. Antreich. GORDIAN: VLSI placement by quadratic programming and slicing optimization. IEEE Transactions on ComputerAided Design, CAD-10:356-365, March 1991.

[15] K. W. Lee and C. Sechen. A new global router for rowbased layout. In Proceedings of the IEEE International Conference on Computer Aided Design, pages 180-183, November 1988.

[16] S. Mayrhofer, M. Pedram, and U. Lauther. A flow-based approach to the placement of boolean networks. In IFIP International Conference on Very Large Scale Integration, 1991.

[17] C. R. Morrison, R. M. Jacoby, and G. D. Hachtel. TECHMAP: Technology mapping with delay and area optimization. In Proceedings of the International Workshop on Logic and Architecture Synthesis for Silicon Compilers, pages 5364, Grenoble, France, May 1988.

[18] M. Pedram. An integrated approach to logic synthesis and physical design. $\mathrm{PhD}$ thesis, University of California, Berkeley, August 1991. Technical Report UCB/ERL M91/69.

[19] M. Pedram and N. Bhat. Layout driven logic restructuring/ decomposition. In Proceedings of the IEEE International Conference on Computer Aided Design, pages 134-137, November 1991.

[20] M. Pedram and N. Bhat. Layout driven technology mapping. In Proceedings of the 28th Design Automation Conference, pages 99-105, June 1991.

[21] J. Reed, A. Sangiovanni-Vincentelli, and M. Santamauro. A new symbolic channel router: YACR2. IEEE Trans. on Computer-Aided Design, 4(3):208-219, March 1985.

[22] T. Sakurai. Approximation of wiring delays in MOSFET LSI. IEEE Journal of Solid State Circuits, SC-18(4):418426, August 1983.

[23] K. C. Saraswat and F. Mohammadi. Effect of scaling of interconnections on the time delay of VLSI circuits. IEEE Transactions on Electron Devices, ED-29:645-650, 1982.

[24] H. Savoj and H. Y. Wang. Improved scripts in MIS-II for logic minimization of combinational circuits. In Proceedings of the International Workshop on Logic Synthesis, May 1991.

[25] K. J. Singh, A. Wang, R. K. Brayton, and A. SangiovanniVincentelli. Timing optimization of combinational logic. In Proceedings of the IEEE International Conference on Computer Aided Design, pages 282-285, November 1988.

[26] H. J. Touati, H. Savoj, and R. K. Brayton. Delay optimization of combinational logic circuits by clustering and partial collapsing. In Proceedings of the IEEE International Conference on Computer Aided Design, pages 188-191, November 1991.

\section{Authors' Biographies}

Massoud Pedram is an associate professor of Electrical Engineering-Systems at the University of South- ern California. He received his B.S. degree in Electrical Engineering from the California Institute of Technology in 1986 and his M.S. and Ph.D. degrees in Electrical Engineering and Computer Sciences from the University of California, Berkeley in 1989 and 1991, respectively.

Dr. Pedram received best paper awards at the IEEE Int'l Conf. on Computer Design (1989), and the ACM/IEEE Design Automation Conference (1996) and the National Science Foundation's Young Investigator Award (1994). He has served on the Technical Program Committee of the ACM/IEEE Design Automation Conference since 1992 and is the co-founder and general chair of the International Symposium on Low Power Design (1995) and the technical co-chair of the International Symposium on Low Power Electronics and Design (1996). His research interests and contributions have been in the areas of logic synthesis, physical design and CAD for low power.

Narasimha B. Bhat received the B.E. degree in Electronics and Communication from the Manipal Institute of Technology (Mangalore University), India, in 1988, the M.E. degree in Electrical Communication Engineering from the Indian Institute of Science, Bangalore, in 1989, and the Ph.D. degree in Electrical Engineering from the University of California, Berkeley, in 1993.

Dr. Bhat is currently a Member of the Technical Staff at Synopsys, Inc. Mountain View, CA. He was a Teaching Fellow at the Manipal Institute of Technology, India, in 1989. From 1989 to 1993 he was a Research Assistant in the Electronics Research Laboratory at U.C. Berkeley. He then worked at AT\&T Bell Laboratories, Murray Hill, NJ from 1993 to 1995. His research interests include FPGAs (CAD and architecture), logic synthesis, hardware accelerators and neural nets.

Ernest S. Kuh is the William S. Floyd, Jr. Professor Emeritus in Engineering of the Department of Electrical Engineering at the University of California, Berkeley. He joined the EECS Department faculty in 1956. From 1968 to 1972 he served as chair of the department; from 1973 to 1980 he served as Dean of the College of Engineering. From 1952 to 1956 he was a member of the Technical Staff at Bell Telephone Laboratories in Murray Hill, New Jersey. 
Prof. Kuh attended Shanghai Jiao Tong University from 1945 to 1947; received the B.S. degree from the University of Michigan, Ann Arbor, in 1949; the S.M. degree from the Massachusetts Institute of Technology, Cambridge, in 1950; and the Ph.D. degree from Stanford University, Stanford, California, in 1952.
Prof. Kuh is a member of the National Academy of Engineering and the Academia Sinica. He is a Fellow of IEEE and AAAS. He has received numerous awards and honors, including the IEEE Centennial Medal, the IEEE Education Medal, and the IEEE Circuits and Systems Society Award. 

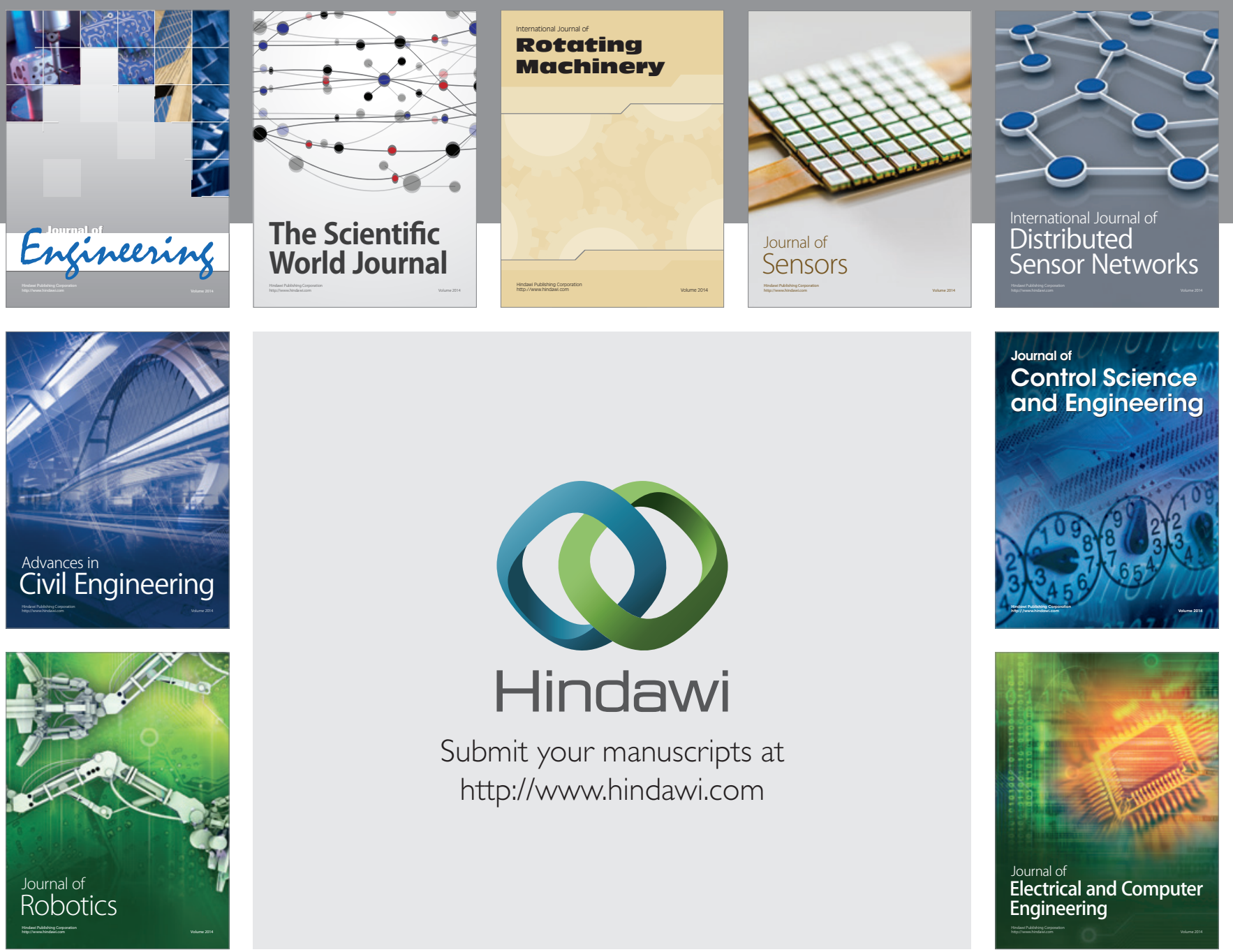

Submit your manuscripts at

http://www.hindawi.com
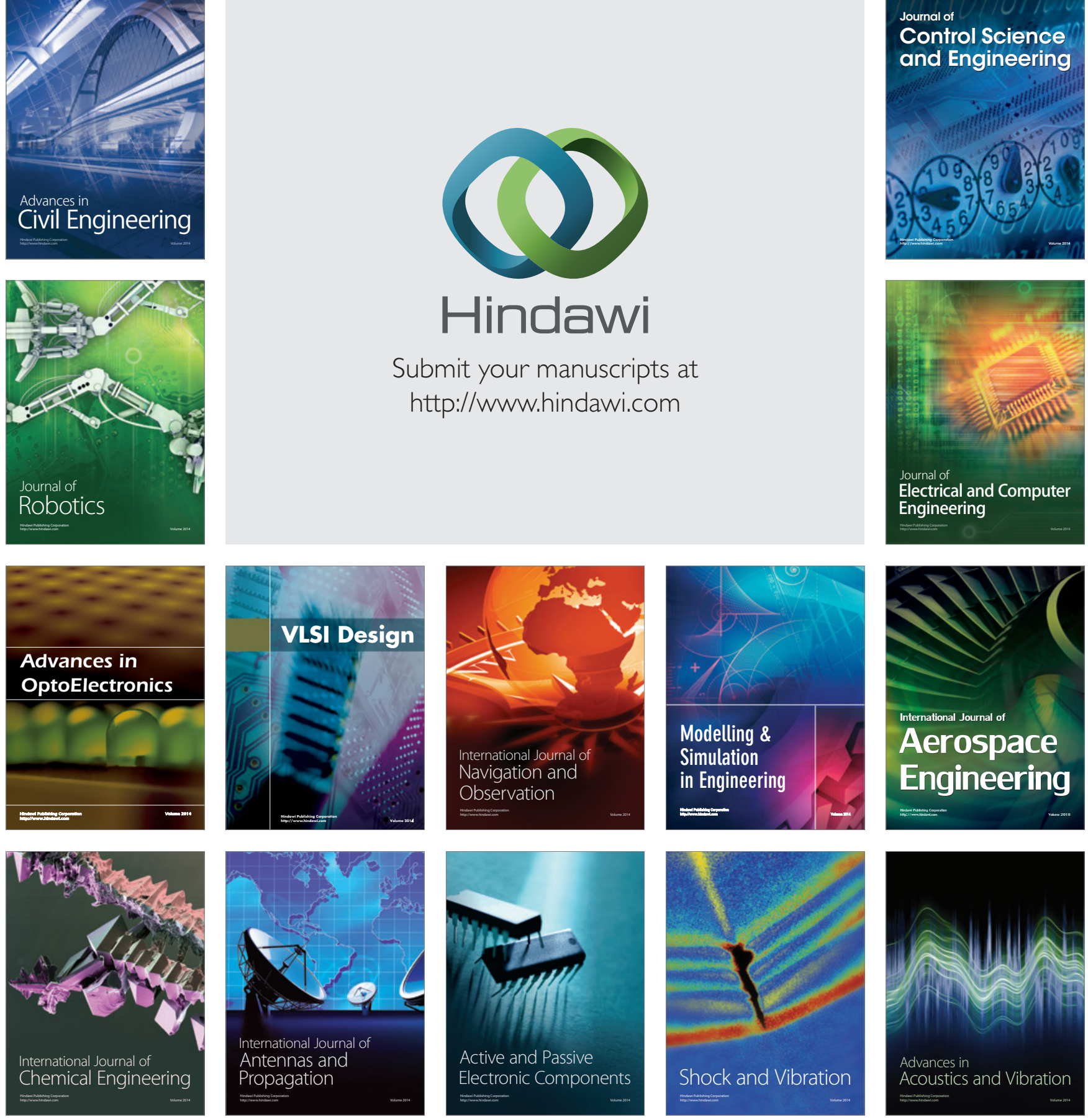